\title{
PHASE SPACE HOLOGRAPHY WITH NO STRINGS ATTACHED
}

\author{
D.V. Khveshchenko \\ Department of Physics and Astronomy, University of North Carolina, Chapel Hill, NC 27599, U. S. A. \\ Email: khvesh@physics.unc.edu
}

Received 20 February 2021; accepted 15 April 2021

\begin{abstract}
We discuss the Wigner function representation from the novel standpoint of establishing a natural holographylike correspondence between the descriptions of a generic quantum system in the phase space ('bulk') picture versus its spacetime ('boundary') counterpart. In certain cases, the former may reduce to the gravity-like dynamics of a local metric-type variable while the latter takes on the form of some bosonized collective field hydrodynamics. This generic pseudo-holographic duality neither relies on any particular symmetry of the system in question, nor does it require any relation to some underlying 'string theory', thus providing a systematic way of constructing practical - as opposed to the previous 'ad hoc' - examples of genuine holographic duality.
\end{abstract}

Keywords: phase space, holographic correspondence, hydrodynamics, effective metric

\section{Generalized holography}

Among all the remarkable theoretical advances under the hashtag \#holography one of the greatest interest to condensed matter physics is the ongoing quest for generalizing the original, highly constrained (i.e. Lorentz- and maximally supersymmetric, etc.), string-theoretical holographic conjecture to as broad as possible (i.e. non-Lorentz-, non-isotropic-, non-supersymmetric, etc.) variety of quantum many-body systems [1-7].

In that regard, the so-called 'bottom-up' holography has been portraying itself as a powerful technique offering solutions to the traditionally hard condensed matter problems. This innovative approach (sometimes referred to as the anti-de-Sitter/condensed matter theory, or AdS/CMT, correspondence) borrows its formal structure and mathematical apparatus (often 'ad verbum', for the lack of an alternative) from the very specific, carefully crafted and highly symmetric examples of such duality known under the acronym AdS/CFT and conjectured in the original context of fundamental string theory and its various reductions $[1-7$.
So far, however, all the attempts of putting applied holography on a firm foundation - either along the lines of the geometrized renormalization group (RG) flow or entanglement dynamics in tensor networks, or by using artificial thermodynamic (Fisher-Ruppeiner, Fubini-Study, etc.) metrics, or else - have remained consistently inconclusive.

Nonetheless, instead of striving to deliver a solid proof of principle, the field of AdS/CMT has, by and large, stayed the course reminiscent of hacker's code cracking: that is, trying to guess some higher-dimensional enhanced gravity-like theory (often on the sole basis of technical convenience) and then rely on the persuasive power of visual agreement between some pre-selected experimental data plots and the (for the most part, numerical) calculations based on the above AdS/CMT 'dictionary'. A great many number of the customarily verbose and frustratingly lookalike accounts of such pursuits can be readily found in the applied holographic literature from the year 2007 onwards [8]. Judging by the factual outcome of this massive attack, though, the code does not appear to have been cracked yet. 
Such fundamental shortcomings notwithstanding, the still continuing occasional exercises in the holographic phenomenology utilize a handful of the popular bulk geometries, especially focusing on the legacy solutions of the prototypical Einstein-Maxwell-dilaton theory. Other than their relative simplicity and sheer availability (alongside, possibly, some lingering anthropic factor) a proper justification of such choices does not appear to have been an essential part of the holographic agenda, regardless of whether the results were meant to be applied to the lattice Hubbard-like models, superconducting cuprates, low-density 2DEG, Dirac/ Weyl materials such as graphene, or else.

Lately, though, a gradual decline in such 'orthodox' applications of the AdS/CMT machinery has been giving way to advanced hydrodynamics of strongly coupled quantum matter and general out-of-equilibrium physics (eigenstate thermalization, many-body (de)localization, chaos spreading, operator growth, etc.). Correspondingly, instead of the once ubiquitous renditions of esoteric black holes, nowadays a typical slide show on the topic of $\mathrm{AdS} / \mathrm{CMT}$ is more likely to feature the images of water flows, rapids, whirlpools, and other familiar hydrodynamic patterns [9].

Of course, hydrodynamics, while suggesting some intriguing holographic connections, has long been discussed outside of any holographic context. Therefore, the renewed appreciation and novel applications (thanks to a number of recent experimental advances) of the theory as earthly as hydrodynamics alone do not provide an answer to the question that should have (but does not seem to have been) long dominated the holographic discourse, that is: 'So why, on Earth, strings?!'.

The goal of this note is to recall a decades-old theoretical approach known as collective field theory [10-23] and its more recent developments that might be capable of providing a much-needed justification for the 'stringy hydrodynamics' (especially, in those non-relativistic and/or rotationally-noninvariant settings that are typical of the condensed matter applications but do not normally occur in the original string-theoretical context). Alternatively, this approach can be viewed as a variant of the long-pursued idea of (non-linear) 'bosonization' that aims to reformulate a quantum theory of interest in terms of some intrinsically geometric bosonic variables.
Notably, while manifesting some features reminiscent of the desired holographic correspondence, this approach demonstrates that the pertinent spacetime metrics may not be chosen at will and are often quite different from the routinely utilized ones.

\section{Phase space quantization}

A systematic description of many-body dynamics in $d$ spatial dimensions calls for the use of the Wigner function $w(\mathbf{x}, \mathbf{p}, t)$ defined in the $2 d+1$-dimensional phase space (plus time). The space of such functions is equipped with the Moyal product

$$
f(\mathbf{x}, \mathbf{p}) * g(\mathbf{x}, \mathbf{p})=f(\mathbf{x}, \mathbf{p}) \mathrm{e}^{\mathrm{i} \frac{\hbar}{2}\left(\overline{\mathrm{o}}_{\mathbf{x}} \overrightarrow{\hat{p}}_{\mathbf{p}}-\bar{\partial}_{\mathbf{p}} \vec{\partial}_{\mathbf{x}}\right)} g(\mathbf{x}, \mathbf{p})
$$

which introduces the symplectic structure through the Moyal bracket

$$
\{f, g\}_{\mathrm{MB}}=f \star g-g \star f .
$$

The Wigner's description is well suited for taking into account the underlying theory's invariance under the phase space volume-preserving diffeomorphisms, including its natural time evolution that can be thought of as a canonical transformation governed by the Liouville theorem.

Furthermore, when quantizing the system via the method of functional integration, the function $w(\mathbf{x}, \mathbf{p}, t)$ becomes a constrained field variable implementing a coadjoint orbit's quantization of the phase space volume-preserving diffeomorphisms à la Kirillov-Kostant.

In this procedure, an orbit's element $\hat{g}\left|\Psi_{0}\right\rangle\left\langle\Psi_{0}\right| \hat{g}^{-1}$ is constructed by acting with an element $\hat{g}$ of the infinite-dimensional group of diffeomorphisms on the projector to a chosen (e.g. ground) state $\left|\Psi_{0}\right\rangle$. In the partition function $Z=\int \mathrm{D} w \exp (-S(w))$ the integration then runs over the functions satisfying the constraints

$$
w \star w=w, \quad \operatorname{Tr} w=1
$$

and governed by the action

$$
S(w)=\int \mathrm{d} x \mathrm{~d} p \mathrm{~d} t\left(\mathrm{i} \int_{0}^{1} \mathrm{~d} s w\left\{\partial_{\tau} w, \partial_{s} w\right\}_{\mathrm{MB}}-w \mathrm{H}\right),
$$

where the first term represents the Berry phase, with the integral over the auxiliary variable $s$ depending 
only on the boundary value $w(\mathbf{x}, \mathbf{p}, t, s=1)=w(\mathbf{x}$, $\mathbf{p}, t)$ at $s=1$. This way, one arrives at the formally exact geometrized description of the non-linear $\sigma$-model type [10-23].

The equation of motion derived from Eq. (4)

$$
\dot{w}+\{w, H\}_{\mathrm{MB}}=0
$$

reproduces the standard kinetic equation when the Moyal bracket is approximated, to the lowest order in the powers of $\hbar$, by the Poisson one (hereafter the dot and prime stand for the time and space derivatives, respectively)

$$
\dot{w}+w^{\prime} \partial_{\mathbf{p}} H_{1}-H_{1}^{\prime} \partial_{\mathbf{p}} w=S t[w]
$$

where the one-body Hamiltonian $H_{1}$ may include an external potential. For example, in the so-called 'noncritical $2 d$ string theory', where the spatial coordinate originates from the eigenvalues of $N \times N$ matrices, it happens to be the inverted oscillator $\left(V \sim-x^{2}\right)[10-$ 23. Also, the $n \geq 2$-body terms $H_{n}$ in the Hamiltonian involving higher powers of $w$ are bundled into the collision integral in the right-hand side.

In the case of fermions, the semiclassical vacuum configuration corresponding to the uniform Fermi sea is described by the expression

$$
w_{0}(\mathbf{x}, \mathbf{p}, t)=\theta\left(\mu(\mathbf{x}, t)-\epsilon_{\mathbf{p}}\right),
$$

where the local chemical potential $\mu(\mathbf{x}, t)$ denotes a sharp boundary between the occupied and vacant momentum eigenstates with the dispersion $\epsilon_{\mathrm{p}}$.

Facilitating further progress with the $2 d+1$-dimensional 'bulk' theory (4) requires a convenient parameterization of the bounding momentum. Previously, a similar task was tackled in the early works on multi-dimensional bosonization, where this goal was achieved by distinguishing between the Fermi momentum $\mathbf{p}_{\mathrm{F}}$ tracing the fiducial Fermi surface (FS) and the normal to the FS ('radial') degree of freedom describing fluctuations of the momentum distribution [24-40.

For instance, in the much studied $d=2$ case the simplified action for the vector $\mathbf{p}_{\mathbf{F}}$ reads

$$
S\left(\mathbf{p}_{\mathbf{F}}\right)=\int \mathrm{d} x \mathrm{~d} t\left(i \int_{0}^{1} \mathrm{~d} s \mathbf{p}_{\mathbf{F}} \partial_{\tau} \mathbf{p}_{\mathbf{F}} \times \partial_{s} \mathbf{p}_{\mathbf{F}}-H\left(\mathbf{p}_{\mathbf{F}}\right)\right),
$$

where the Hamiltonian $H$ is cast in terms of the local density $\rho=\mathbf{p}_{\mathrm{F}} \times \partial_{t} \mathbf{p}_{\mathrm{F}} / 2$.
In what follows, we focus on the case of $d=1$, where the fluctuating FS can be described in terms of $M \geq 1$ pairs of the Fermi momenta $p_{ \pm}^{(n)}(x, t)$ bounding the occupied states $(M>1$ accounts for the possibility of 'folds' in the presence of shock waves and other FS singularities [10-23]).

In particular, the $1 d$ configuration (7) reads

$$
w_{0}(x, p, t)=\sum_{ \pm} \sum_{\alpha=1}^{M}( \pm) \theta\left(p_{ \pm}^{(\alpha)}(x, t)-p\right),
$$

while its small perturbation

$$
\delta w(x, p, t)=\hbar \delta\left(x-x_{\mathrm{cl}}\right) \delta\left(p-p_{\mathrm{cl}}\right)
$$

is strongly peaked at the classical phase space trajectory $\left(x_{c 1}\left(x_{0}, p_{0}, t\right), p_{c 1}\left(x_{0}, p_{0}, t\right)\right)$, where the initial data $x_{0}$ and $p_{0}$ are determined by the current values $(x, p)$ at a later time $t$.

It is worth mentioning, though, that while being capable of faithfully reproducing the long-distance, late-time asymptotics of the response functions, in its practical (hence, approximate) form the $d>1$-dimensional bosonization technique is not well suited for computing the Lindhard-type $2 p_{F}$-singularities, just as it may not be sufficient for the single-particle propagators [24-40].

In $d=1$, despite several decades of studies there has been a recent surge of renewed interest in the out-of-equilibrium dynamics of quantum interacting bosons and fermions. Many of those studies focus on the integrable and non-ergodic systems which are governed by the generalized Gibbs ensembles (GGE) and may not comply with the more generic eigenstate thermalization hypothesis (ETH) [41-67].

It is worth noting that in $1 d$ Eq. (2) represents a classical analog of the infinite-dimensional quantum algebra $W_{\infty}$ composed of the operators $\hat{W}_{m n}=(\hat{x})^{m}(\hat{p})^{n}$ with the commutation relations

$$
\begin{aligned}
& {\left[\hat{W}_{m n}, \hat{W}_{r s}\right]=\sum_{k=1} \frac{(-\hbar)^{k}}{k} \times} \\
& \left(\frac{n ! r !}{(n-k) !(r-k) !}-\frac{m ! s !}{(n-k) !(s-k) !}\right) \times \\
& \hat{W}_{m+r-k, n+s-k},
\end{aligned}
$$

where the r.h.s. reduces to $(m s-n r) \hat{W}_{\mathrm{m}+\mathrm{r}-1, \mathrm{n}+s-1}$ in the $\hbar \rightarrow 0$ limit, thereby encompassing the algebra 
$S L(2, R)$. This algebra has been extensively studied in the context of Quantum Hall Effect (QHE) and the various reincarnations of (effectively) noncommutative spacetimes.

An abstract Hilbert space can be readily equipped with a geometric structure that has long been elucidated alongside the more familiar Berry phase. However, the even (Fubini-Study metric) - as opposed to the odd (Berry curvature) - component of the same rank-2 tensor has been receiving less attention.

Such a phase space metric can be naturally introduced in the context of special coherent - (de) localized neither in the coordinate nor momentum space - states $|p, x, 0\rangle=\mathrm{e}^{\mathrm{i} \hat{P} x-\mathrm{i} \hat{Q} p}|0\rangle$ which minimize both the coordinate and momentum uncertainties.

Allowing, for the sake of generality, some coordinate-momentum cross-correlations, the corresponding Wigner function reads

$$
\begin{aligned}
& w_{\text {coh }}(x, p, 0)=\int \mathrm{d} y \mathrm{e}^{\mathrm{i} p y}\langle\Psi \mid x+y / 2\rangle\langle x-y / 2 \mid \Psi\rangle \\
& =\frac{\hbar}{D^{1 / 2}} \exp \left(-\frac{\sigma_{p} \delta x^{2}+\sigma_{x} \delta p^{2}+2 \sigma_{x p} \delta x \delta p}{2 D}\right),
\end{aligned}
$$

where $\delta x=x-x_{0}, \delta p=p-p_{0}, D=\sigma_{x} \sigma_{p}-\sigma_{x p}^{2}$, and the parameters $\sigma_{x}, \sigma_{p}, \sigma_{x p}$ control the Gaussian coordinate and/or momentum variations.

The above suggests a naturally defined Fubinitype metric on the phase space [68-78]

$$
\mathrm{d} s^{2}=\left(\left\langle\partial_{\mu} \Psi \mid \partial_{v} \Psi\right\rangle-\left\langle\Psi \mid \partial_{\mu} \Psi\right\rangle\left\langle\partial_{\nu} \Psi \mid \Psi\right\rangle\right) \delta_{\mu} \delta_{v},
$$

where $\delta_{\mu}=(\mathrm{d} x, \mathrm{~d} p)$. Taken at its face value, this formula establishes some form of superficial correspondence between single-particle quantum mechanics and $2 d$ metrics that can be viewed as solutions of certain classical gravity.

Further generalizing Eq. (13) to include energy fluctuations one arrives at the (Euclidean) $3 d$ metric

$$
\begin{aligned}
& \mathrm{d} s^{2}=\left\langle(\Delta \hat{H})^{2}\right\rangle \mathrm{d} t^{2}+\left\langle(\Delta \hat{x})^{2}\right\rangle \mathrm{d} p^{2} \\
& +\left\langle(\Delta \hat{p})^{2}\right\rangle \mathrm{d} x^{2}+2\langle\Delta \hat{x} \Delta \hat{p}\rangle \mathrm{d} x \mathrm{~d} p
\end{aligned}
$$

given by the uncertainties of the conjugate variables $(x \leftrightarrow p, t \leftrightarrow H)$. Also, considering the metric (14) to be the expectation value $\mathrm{d} s^{2}=\left\langle\Psi\left|\mathrm{d} \hat{s}^{2}\right| \Psi\right\rangle$ of the operator-valued interval $\mathrm{d} \hat{s}^{2}$ paves the way for promoting the bulk (phase space)-to-boundary (spacetime) relationship to the quantum level.
As the operators' uncertainties depend on the choice of the state $|\Psi\rangle$, so does the dual metric (14). Heuristically, one might expect that for the single-particle dispersion governed by the dynamical exponent $z\left(\epsilon_{\mathbf{p}} \sim \mathbf{p}^{z}\right)$ the above variances behave as follows,

$$
\begin{aligned}
& \left\langle(\Delta p)^{2}\right\rangle \sim\left\langle(\Delta x)^{2}\right\rangle^{-1} \sim p^{2}, \\
& \langle(\Delta x)(\Delta p)\rangle \sim\langle(\Delta p)\rangle^{1 / 2}\left\langle(\Delta p)^{-1 / 2}\right\rangle \sim p^{0}, \\
& \left\langle(\Delta H)^{2}\right\rangle \sim\left\langle(\Delta p)^{2}\right\rangle^{z} \sim p^{2 z},
\end{aligned}
$$

so that the metric (14) conforms to the so-called Lifshitz variety (coefficients $A, B, C$ and $D$ are constants)

$$
\mathrm{d} s^{2}=A p^{2 z} \mathrm{~d} t^{2}+B p^{2} \mathrm{~d} x^{2}+C \frac{\mathrm{d} p^{2}}{p^{2}}+2 D \mathrm{~d} x \mathrm{~d} p,
$$

which has been often invoked in various applications of AdS/CMT [1-7].

\section{Non-linear hydrodynamics}

The formally exact representation (4) of the phase space dynamics provides a basis for further simplifications, thus giving rise to (semi)classical hydrodynamic equations for the various moments of the Wigner distribution

$$
w_{n}(\mathbf{x}, t)=\int \mathrm{d} \mathbf{p} w(\mathbf{x}, \mathbf{p}, t) \mathbf{p}^{n} .
$$

Among those moments are such standard hydrodynamic variables as the local mass $\rho(n=0)$, momentum $Q(n=1)$, and energy $\epsilon(n=z)$ densities, respectively. This transition from the entire Wigner function to the first few of its moments can be thought of as a dimensional reduction from the $2 d+1$-dimensional bulk (phase space) to its $d+1$-dimensional boundary hyper-surface (spacetime) which, in practice, amounts to mere integration over the $d$-dimensional momentum.

Correspondingly, the mass $J_{\rho}$, momentum $J_{Q}$, and energy $J_{\epsilon}$ currents are given by the general expression

$$
\mathbf{J}_{n}=\int \mathrm{d} \mathbf{p} \frac{\partial_{\epsilon_{p}}}{\partial \mathbf{p}} w(\mathbf{x}, \mathbf{p}, t) \mathbf{p}^{n}
$$

for $n=0,1,2$. 
In the case of $d=1$, the lowest moments of the Wigner function (17) correspond to the aforementioned bounding Fermi momenta $p_{ \pm}=\int \mathrm{d} p w(1 \pm \operatorname{sgn} p) / 2$ or, equivalently, the local density and material velocity

$$
\rho=\frac{1}{2 \pi}\left(p_{+}-p_{-}\right), \quad v=\frac{1}{2}\left(p_{+}+p_{-}\right) .
$$

Note that limiting the momentum values to the interval $0<p<\infty$, similar to the holographic radial variable, is dictated by the chiral nature of the excitations carrying sign-definite momenta.

These variables have the Poisson bracket

$$
\{\rho(x), v(y)\}=\partial_{x} \delta(x-y) .
$$

Then taking the various moments of Eq. (5) one arrives at the hydrodynamic equations of motion which include the continuity equation

$$
\dot{\rho}+(\rho v)^{\prime}=0
$$

and the inviscid Navier-Stokes (a.k.a. Euler/Burgers/Hopf) one

$$
\dot{v}+v v^{\prime}=-\frac{P^{\prime}}{\rho}-\kappa \frac{V^{\prime}}{\rho},
$$

where the 'quantum pressure' $P(\rho)$ (internal stress tensor of the $1 d$ quantum fluid) is a system-specific function of the local density, while the last term with the dispersion curvature $\kappa=\partial_{p}{ }^{2} \epsilon_{p}$ represents the force exerted by the external potential (if any).

It is well known that the non-linear hydrodynamic Eqs. (21) and (22) can even be derived from the Schroedinger equation for a free particle of mass $m$ [68-78]. Specifically, by applying the Madelung parameterization of the wave function $\Psi(x, t)=\sqrt{\rho(x, t)} \mathrm{e}^{\mathrm{i}(x, t)}$ and separating out the real and imaginary parts one arrives at the coupled continuity and Navier-Stokes equations, respectively, where

$$
v=-\frac{1}{2 \mathrm{i} m}\left(\frac{\Psi^{*}}{\Psi}\right)^{\prime}, \quad \rho=|\Psi|^{2} .
$$

In the r.h.s. of Eq. (22) the pressure

$$
P=-\frac{\hbar^{2}}{8 m} \frac{\left(\rho^{\prime}\right)^{2}}{\rho}
$$

contributes towards the overall energy density $\epsilon=\rho v^{2} / 2+P$ which, in general, might be neither polynomial nor separable as a sum of two chiral components $P_{ \pm}\left(p_{ \pm}\right)$.

It appears, however, that the pressure gradient term couples excitations with opposite chiralities (left/right moving) at the level of operators with dimensions of four or greater. Moreover, even if present, the non-chiral corrections do not affect the states which are composed exclusively of the chiral excitations with $p_{+}=0$ or $p_{-}=0$.

Thus, an arbitrary single-particle wave function $\Psi(x, t)=(2 \pi \mathrm{i} \hbar t / m)^{-1 / 2} \int \mathrm{d} y \mathrm{e}^{\mathrm{i} m(x-y)^{2} / 2 \hbar t} \Psi_{0}(y)$ originating from the initial state $\Psi_{0}(x)$ provides a valid solution to the hydrodynamic equations $(21,22)$ with the pressure (24). Correspondingly, the pair of functions $\rho(x, t)$ and $v(x, t)$ determines a certain dual metric, as explained below.

\section{Solvable hierarchies}

For certain choices of the Hamiltonian $H$ Eq. (22) appears to belong to the infinite Korteweg-de Vries $(\mathrm{KdV})$ hierarchy of integrable $1 d$ systems [41-67]. Such Hamiltonians $H_{k}^{ \pm}$are related to the Gelfand-Dickey polynomials and form an infinite set of the integrals of motion in involution $\left(\left[H_{n}^{ \pm}\right.\right.$, $\left.H_{m}^{ \pm}\right]=0$ ). In the asymptotically free regime of large momenta (high energies) the $k$ th member of this family describes small-amplitude excitations with the dispersion exhibiting the dynamical exponent $z=2 k-1$.

In particular, the generic $1 d$ Luttinger liquid (LL) behaviour is associated with the quadratic Hamiltonian $H=\Sigma_{ \pm} H_{1}^{ \pm}$given by the standard Sugawara construction

$$
H_{1}=\frac{1}{4} \sum_{ \pm} p_{ \pm}^{2}=\frac{1}{2}\left(v^{2}+\pi^{2} \rho^{2}\right),
$$

which gives rise to the equation of motion

$$
\dot{p}_{ \pm} \mp p_{\mp}^{\prime}=0
$$

whose solutions given by the (anti)holomorphic functions $p_{ \pm}\left(x_{ \pm}\right)$describe two decoupled chiral $\left(x_{ \pm}=x \pm t\right)$ pseudo-relativistic $(z=1)$ excitations.

Corrections to the LL Hamiltonian (25) may come from both the Gaussian terms of higher operator dimensions which represent non-linear terms in the dispersion of the collective $\rho$ - and $v$-modes, as well as from the non-Gaussian ones which are due to some intrinsic non-linearity of the $1 d$ 
dispersion, as in the case of non-relativistic fermions at a finite density [41-67.

For example, the next (2nd) member of the KdV family is given by the non-Gaussian expression

$$
\begin{aligned}
& H_{2}=\sum_{ \pm}( \pm) \frac{1}{3} p_{ \pm}^{3}+\frac{1}{2}\left(p_{ \pm}^{\prime}\right)^{2}= \\
& \frac{1}{2} \rho v^{2}+\frac{\pi^{2}}{6} \rho^{3}+\frac{1}{2}\left(v^{\prime}\right)^{2}+\frac{\pi^{2}}{2}\left(\rho^{\prime}\right)^{2}
\end{aligned}
$$

for which the chiral components of Eq. (22) still remain uncoupled:

$$
\dot{p}_{ \pm} \pm 3 p_{ \pm} p_{ \pm}^{\prime}+p_{ \pm}^{\prime \prime \prime}=0
$$

In the asymptotic regime of high energies the linearized Eq. (28) describes small waves with the expressly Lorentz-non-invariant cubic dispersion $(z=3)$.

In the opposite, low-energy and essentially nonperturbative, limit Eq. (28) permits non-linear solitonic excitations ('cnoidal' waves) 41-67

$$
v(x, t) \sim \frac{1}{\cosh ^{2} x_{ \pm}}
$$

whose propagation is described by the dispersion $\epsilon_{p} \sim p^{5 / 3}$. Compactifying the spatial coordinate into a finite-length circle would then replace Eq. (29) with the elliptic Jacobi function.

In general, the non-Gaussian terms in the Hamitonian are sensitive to the microscopic details of the model and stem from both kinetic and potential terms in the total energy. Specifically, in the case of hard-core bosons, such as the Tonks-Girardeau limit of the Lieb-Liniger model, the Hamiltonian includes the pressure term $P(\rho) \sim \rho^{3}$. By contrast, in the quantum Toda chain the function $P(\rho)$ is nonpolynomial. However, despite not being dividable into a sum of two chiral terms, the latter can still fit into the KdV Hamiltonian low momenta [41-67].

Likewise, the deviations from the LL regime associated with a finite dispersion curvature and/or chiral interactions can be studied with the use of a linear combination $\hat{H}_{2}+\hat{H}_{2}+\ldots$ of Eqs. (25) and (27). This way, one can obtain non-linear corrections $\delta \epsilon_{p} \sim p^{5 / 3}$ to the linear LL spectrum at small momenta [41-67.

Moreover, Eq. (27) can be further modified by including irrelevant non-Gaussian terms, such as $p_{ \pm}^{4}$, without destroying its integrability. Indeed, such extension results in yet another, Gardner, equation (a.k.a. mixed KdV-m(modified)KdV, the two equations being related by virtue of the Miura transformation $p_{ \pm} \rightarrow p_{ \pm}^{2}+p_{ \pm}^{\prime}$ ).

As a hallmark of integrability, the higher- $k$ level members of the KdV hierarchy possess the bi-Hamiltonian structure relating them as follows [79-95]:

$$
\partial_{x} \frac{\delta H_{k+1}^{ \pm}}{\delta p_{ \pm}}=\mathcal{D}_{x}^{ \pm} \frac{\delta H_{k}^{ \pm}}{\delta p_{ \pm}} .
$$

Here the long derivative is

$$
\mathcal{D}_{x}^{ \pm}=2 p_{ \pm} \partial_{x}+\partial_{x} p_{ \pm}+\partial_{x}^{3} .
$$

The higher- $k$ level members of the KdV and $\mathrm{mKdV}$ families can also be morphed into a twoparameter Gardner sequence of Hamiltonians. Furthermore, certain solvable systems of $M \geq$ coupled non-linear equations were shown to be associated with the higher-spin symmetry algebras $S L(M, R)$ (e.g. the Boussinesq equations for $M=3$ ) [96-101].

Generic Hamiltonians $\Sigma_{k} \mu_{k} H_{k}^{ \pm}$which include different members of the integrable family can be used to derive zero entropy GGE hydrodynamics. In particular, one can study crossovers between the LL and higher- $k$ level regimes at varying momenta. In essence, this construction provides a (formally exact) bosonization scheme that was fully exploited, e.g. in the context of the solvable Calogero-Sutherland model [41-67].

Under the time evolution governed by the superposition of different $H_{n}$ a generic initial condition produces a collection of solitons with different velocities and a continuum of decaying dispersive waves. Being more robust the soliton excitations dominate in the late-time behaviour and, in particular, the system's equilibration towards a steady GGE state described by the density matrix $\hat{\mathcal{G}}=\exp \left(-\sum_{k} \mu_{k}^{ \pm} \hat{H}_{k}^{ \pm}\right)$, where the chemical potentials $\mu_{k}$ are to be determined by equating the averages of the commuting charges $\left\langle\hat{H}_{k}^{ \pm}\right\rangle=\operatorname{Tr}\left(\hat{\mathcal{G}} \hat{H}_{k}^{ \pm}\right) / \operatorname{Tr} \hat{\mathcal{G}}$ to their chosen values.

Along these lines, one can also study the von Neumann entropy $S=-\operatorname{Tr} \hat{\mathcal{G}} \ln \hat{\mathcal{G}}$. The Wigner function satisfying the constraints (3) corresponds to a pure state of zero entropy and the presence of an infinite number of conserved charges precludes standard thermalization. When the constraint 
ceases to hold, the state becomes mixed, thus resulting in a finite entropy. The ensuing thermalization can be accounted for by introducing viscous terms, such as $\eta \rho^{\prime \prime}$, in the r.h.s. of Eq. (22).

\section{Dual bulk gravity}

A deep relationship between classical gravity and hydrodynamics has long been known as one particular take on the holographic paradigm, often referred to as the 'fluid-gravity' correspondence. The crux of the matter is observation of the similarity between the asymptotic near-boundary behaviour of the Einstein equations for the bulk metric and the Navier-Stokes ones describing a dual boundary fluid in one lesser dimension (besides, the complementary hydrodynamic behaviour near the event horizon can be similar to that at the boundary). Albeit being truncated and, therefore, approximate such relations can be systematically improved, thus enabling certain computational simplifications. Whether or not this duality can be promoted to the quantum level requires further analysis.

Remarkably, in the case of $d=1$ this correspondence becomes exact. Specifically, the Einstein equations stemming from the action of $3 d$ gravity with a negative cosmological constant (here $l$ and $G$ are the AdS radius and Newton's constant, respectively)

$$
S=\frac{l}{16 \pi G} \int \mathrm{d} x \mathrm{~d} t \mathrm{~d} p \sqrt{g}\left(R+2 / l^{2}\right)=0
$$

coincide with the equations describing two decoupled Chern-Simons (CS) models with the combined action $79-94$

$$
S=\frac{l}{16 \pi G} \operatorname{Tr} \int \mathrm{d} x \mathrm{~d} p \mathrm{~d} t \epsilon^{\mu \nu \lambda}\left(\hat{A}_{\mu}^{ \pm} \partial_{\nu} \hat{A}_{\lambda}^{ \pm}+\hat{A}_{\mu}^{ \pm} \hat{A}_{v}^{ \pm} \hat{A}_{\lambda}^{ \pm}\right) .
$$

The chiral connections $\hat{A}_{\mu}^{ \pm}$are matrices that can be expanded in the basis spanned by the generators $\hat{L}_{0, \pm 1}^{ \pm}$of the algebra $S L(2, R) \times S L(2, R)=S O(2,2)$. They obey the commutation relations $\left[\hat{L}_{n^{\prime}}^{ \pm}\right.$ $\left.\hat{L}_{m}^{ \pm}\right]=(n-m) \hat{L}_{n+m}^{ \pm}$and are normalized, $\operatorname{Tr} \hat{L}_{n}^{ \pm}$ $\hat{L}_{m}^{ \pm}=1 / 2 \delta_{n 0} \delta_{m 0}-\delta_{n 1} \delta_{m,-1}$.

The topological action (33) then reduces to a pure boundary term while the equation of motion becomes that of null curvature

$$
\partial_{\mu} \hat{A}_{v}^{ \pm}+\hat{A}_{\mu}^{ \pm} \hat{A}_{v}^{ \pm}-(\mu \leftrightarrow v)=0 .
$$

Parameterizing its solutions in terms of an arbitrary group element $\hat{\chi}_{ \pm}$and functions $p_{ \pm}\left(x_{ \pm}\right)$and $\mu_{ \pm}\left(x_{ \pm}\right)$

$$
\hat{A}^{ \pm}(x, p, t)=\hat{\chi}_{ \pm}^{-1}(p) \hat{L}_{0}\left(\mu_{ \pm} \mathrm{d} t \pm p_{ \pm} \mathrm{d} x\right) \hat{\chi}_{ \pm}(p),
$$

one finds this equation to be equivalent to

$$
\dot{p}_{ \pm} \mp \mu_{ \pm}^{\prime}=0,
$$

which, in turn, coincides with one of the above solvable equations, provided that the chemical potentials $\mu_{ \pm}$conjugate to the variables $p_{ \pm}$are given by the derivatives

$$
\mu_{ \pm}=\frac{\delta H^{ \pm}}{\delta p_{ \pm}} .
$$

Choosing the Hamiltonian appropriately one can then reproduce the solvable $(\mathrm{m}) \mathrm{KdV}$, Gardner, and other equations. In particular, the KdV family is recovered for

$$
\begin{aligned}
& \hat{A}_{p}^{ \pm}(x, p, t)=\frac{1}{p} \hat{L}_{0}^{ \pm}, \quad \hat{A}_{x}^{ \pm}(x, p, t)=p \hat{L}_{1}^{ \pm}-\frac{p_{ \pm}}{p} \hat{L}_{-1}^{ \pm}, \\
& \hat{A}_{t}^{ \pm}(x, p, t)=p \mu_{ \pm} \hat{L}_{1}^{ \pm}-\mu_{ \pm}^{\prime} \hat{L}_{0}^{ \pm}+\frac{\mu^{\prime \prime}{ }_{ \pm}-\mu_{ \pm} p_{ \pm}}{2 p} \hat{L}_{-1}^{ \pm} .
\end{aligned}
$$

the expressions that are manifestly Lorentz-noninvariant for all $k>1$.

Moreover, the equation of motion (34) can be converted into that of the gravity model (32) under the identification of the $3 d$ metric

$$
g_{\mu \nu}=\frac{l^{2}}{4}\left\langle\left(A_{\mu}^{+}-A_{\mu}^{-}\right)\left(A_{v}^{+}-A_{v}^{-}\right)\right\rangle .
$$

On the gravity side the different saddle points of the coherent states path integral can be identified as globally distinguishable (but locally $\mathrm{AdS}_{3}$ ) classical solutions. In particular, it can be shown that the only minima of the action (32) corresponding to the boundary Hamiltonian $H_{1}+H_{2}$ are those with a constant (negative) curvature. The two competing minima are the thermal $\mathrm{AdS}_{3}$ and BTZ (Banados-Teitelboim-Zanelli) black hole.

However, by introducing higher order terms $H_{k}$ with $k \geq 3$ one can generate new KdV-charged black hole configurations [79-95]. The corresponding boundary theory is encoded in the boundary conditions for the connection (35), 
by varying which one can explore a variety of the integrable $1 d$ systems.

The standard LL with $k=1$ is reproduced by introducing the original Brown-Henneaux boundary conditions with constant $\mu_{ \pm} \sim p_{ \pm}$, the outer/ inner horizons being located at $p_{>k<}=\left(p_{+} \pm p_{-}\right) / 2$. The dual metric

$$
\begin{aligned}
& \mathrm{d} s^{2}=\frac{\mathrm{d} p^{2}}{p^{2}}+\left(p^{2}-2\left(p_{+}+p_{-}\right)+\frac{p_{+}^{2}+p_{-}^{2}}{p^{2}}\right) \mathrm{d} t^{2} \\
& +\left(p^{2}+2\left(p_{+}+p_{-}\right)+\frac{p_{+}^{2}+p_{-}^{2}}{p^{2}}\right) d x^{2}+\left(p_{+} p_{-}\right) \mathrm{d} x \mathrm{~d} t
\end{aligned}
$$

describes a rotating BTZ black hole with the event horizon but no curvature singularity.

For static, yet non-constant $p_{ \pm}(x)$ the corresponding boundary solutions possess non-trivial global charges given by the chiral surface integrals $H_{k}^{ \pm}$while their bulk counterparts can be regarded as black holes with multi-graviton excitations ('soft hair') [79-95].

The general solution can be obtained by acting on the ground state (e.g. BTZ black hole) with elements of the asymptotic symmetry group commuting with the Hamiltonian. This way one can construct various constant curvature, yet locally AdS spacetimes with an anisotropic Lifshitz scaling and dynamical exponent $z=2 k-1$. This opens up the possibility of studying nonrelativistic holography without the need of bulk geometries which are asymptotically Lifshitz spacetimes. Furthermore, in the case of a higher-spin symmetry $S L(M, R)$ the list of attainable gravitational backgrounds may include the asymptotically Lobachevsky, Schroedinger, warped AdS, etc. spacetimes [102-110].

Shocks and other abrupt perturbations are characterized by FS breakdowns and emergence of folds where the spatial derivative $\rho$ ' diverges, thereby requiring several pairs of the bounding momenta $p_{ \pm}$. In the presence of shocks, the conventional spacetime hydrodynamics becomes insufficient for describing long-time behaviour, although the fullfledged phase space hydrodynamics can avoid such problems.

In that regard, particularly interesting are the non-stationary configurations representing particles released from a confining potential which gets suddenly switched on/off [111-113].
Such quenching profiles generically have spacelike boundaries where the saddle point solutions of the collective field hydrodynamics diverge at finite times and the semi-classical description fails. Ascertaining the emergent spacetimes and their dynamics then requires a detailed study of fluctuations around the pertinent saddle points.

\section{Reductions and generalizations}

Despite having been repeatedly stated and extensively analyzed at the level of salient symmetries and concomitant algebraic properties, the general gravity/fluid correspondence in dimensions $d>1$ has not yet been put to much of a practical use [110. 114-125.

Specifically, such a relationship was shown to exist between the solutions of classical $d+2$-dimensional gravity and their $d+1$-dimensional hydrodynamic counterparts, whereby the former would be given by the metric

$$
\mathrm{d} s^{2}=\frac{\mathrm{d} p^{2}}{f(p) p^{2}}+p^{2}\left(\Delta_{\mu \nu}-f(p) u_{\mu} u_{\nu}\right) \mathrm{d} x^{\mu} \mathrm{d} x^{v},
$$

parameterized in terms of the spacetime-dependent co-variant velocity $u_{\mu}(\mathbf{x}, t)$ and local temperature $T(\mathbf{x}, t)$ 110, 114-125]. The latter satisfy the hydrodynamic equations on a fixed background, provided that $f(p)=1(4 \pi T / p)^{d}$ and $\Delta_{\mu \nu}=g_{\mu \nu}+u_{\mu} u_{v}$. Thus, a given fluid profile can be associated with a certain asymptotically $\mathrm{AdS}_{d+2}$-like spacetime with a horizon located at $p_{h}=4 \pi T$.

A still more general (asymptotically accurate) solution can be constructed with the use of the metric ansatz

$$
g_{\mu v}(\mathbf{x}, p, t)=\frac{g_{\mu \nu}^{(0)}}{p^{2}}+g_{\mu \nu}^{(2)}+\frac{p^{2}}{4} g^{(2)} g^{(0)} g_{\mu \nu}^{(2)},
$$

with arbitrary functions $g_{\mu \nu}^{(0,2)}(\mathbf{x}, t)$ 110, 114-125.

In contrast to the generic case of $d>1$, pure gravity in $d \leq 1$ does not support any finite energy excitations. Therefore, the fluid/gravity correspondence takes on a particularly simple form where the dual bulk theory appears to be non-dynamical and fully determined by the gapless boundary degrees of freedom ('boundary gravitons').

Of course, such a scenario of 'holography light' does not quite rise to the level of genuine holographic correspondence where the bulk theory is 
supposed to have a non-trivial quantum dynamics which gets quenched and turns classical only in a certain (large $N$ ) limit. It should be noted, however, that, barring a few exceptions, the customarily assumed 'classicality' of the bulk geometry (regardless of whether or not the $1 / \mathrm{N}$ - and/or 'stringy' corrections are important) and a complete neglect of any back-reaction on the fixed background metric appear to be by far the most common approximations routinely made in the absolute majority of all the AdS/CMT calculations performed so far [1-8].

Nonetheless, there are still important differences between the situations in $d=0$ and $d=1$. As per the above discussion, the latter is described by the LL action of two chiral $1 d$ bosons $\phi_{ \pm}\left(x_{ \pm}\right)=\ln \Phi_{ \pm}^{\prime}\left(x_{ \pm}\right)$

$$
\begin{aligned}
S_{L L}^{ \pm} & =\int \mathrm{d} x \mathrm{~d} t \frac{\Phi_{ \pm}^{\prime \prime}\left(\dot{\Phi}_{ \pm}^{\prime} \mp \Phi_{ \pm}^{\prime \prime}\right)}{\left(\Phi_{ \pm}^{\prime \prime}\right)^{2}} \\
& =\int \mathrm{d} x \mathrm{~d} t \phi_{ \pm}^{\prime}\left(\dot{\phi}_{ \pm} \mp \phi_{ \pm}^{\prime}\right) .
\end{aligned}
$$

This action can also be obtained from the more general Alekseev-Shatashvili functional which performs path-integral quantization on the co-adjoint orbit of the (double) Virasoro group. Alternatively, it can be identified with the large central charge limit in the conformal Liouville model, thus relating the latter to its namesake (Liouville) theorem governing the phase space dynamics in the (semi) classical limit.

Besides, this action can be viewed as a complexity functional defining an associated quantum-information type of geometry on the Virasoro group, its lower bound being given by the length of a proper geodesic on the co-adjoint orbit [126-131].

In turn, the extensively studied case of $d=0$ can be attained in the $\mathrm{AdS}_{3}$ theory by taking the limit of a vanishing length of the compactified spatial dimension. The resulting $\mathrm{AdS}_{2}$ bulk theory, as well as its JT (Jackiw-Teitelboim) extension, support a pseudo-Goldstone time reparameterization mode with the $1 d$ boundary action given by the Schwarzian derivative [132-170].

Equivalently, it can be cast in terms of the Liouville quantum mechanics on the quotient $\operatorname{Diff}\left(S^{1}\right) /$ $\operatorname{PSL}(2, R)$ with the action

$$
S_{L}=\int \mathrm{d} t\left(\frac{1}{2} \dot{\phi}^{2}+\lambda \mathrm{e}^{\phi}\right)
$$

for $\phi(t)=\ln \dot{\Phi}(t)$, where $t \rightarrow \Phi(t)$ is a diffeomorphism of the thermal circle. In the context of the space-less random SYK (Sachdev-Ye-Kitaev) and non-random tensor models this orbit emerges as the result of factoring out the subspace of zero modes reflective of the $S L(2, R)$ symmetry of the conformal saddle-point solutions [132-170]. The integrable $1 d$ dynamics in such models is spatially ultra-local and corresponds to $z=\infty$, thus being reminiscent of the popular AdS/CMT schemes [1-7].

Notably, in contrast to the marginal nature of the $1 d$ LL theory where the interaction remains important at all energy/temperature scales, in the SYK tensor models it is strongly relevant in the infrared, thus only affecting the conformal mean-field solutions below a certain energy/temperature scale. Also, the maximally chaotic AdS $/ J T$ gravity can be dual not to a certain quantum mechanical $(d=0)$ theory but (as in the case of SYK) a random ensemble thereof. For comparison, in $d=1$ neither the boundary theory (43) saturates the chaos bound, nor is the bulk behaviour dominated by pure gravity.

In practice, establishing the SYK-to-AdS ${ }_{2}$ duality involves matching thermodynamic properties of the two systems, alongside their various correlation functions. However, achieving this correspondence beyond the lowest order (two-point) correlation functions requires one to introduce additional 'matter' fields in the bulk which represents a tower of higher-spin operators with the anomalous dimensions that all scale comparably with $1 / l[132-$ 170.

Likewise, in the $\mathrm{KdV}$-to- $\mathrm{AdS}_{3}$ correspondence the entropy, free energy, etc. can be matched as well, giving rise to the dependences $S=\pi / 4 \sum_{ \pm} p_{ \pm}^{d} \sim T^{1 / z}$ and $E=\sum_{ \pm}\left\langle\hat{H}_{k}^{ \pm}\right\rangle \sim T^{1+1 / z}$, provided that one chooses $\mu_{ \pm} \sim T$ in order for the metrics (39) to remain regular everywhere in space. Notably, the thermodynamics of $\mathrm{KdV}$-charged black holes differs from that of the usual BTZ ones [79-95.

Comparison between the pertinent microstates on both sides of the latter correspondence relies on the fact that the $2 \mathrm{~d}$ phase space can be spanned by the overcomplete basis of coherent states $|\Psi\rangle=\exp \left(\mathrm{i} \sum_{n m} c_{n m} \hat{W}_{n m}\right)|0\rangle$ while the boundary $1 \mathrm{~d}$ theory operates in the Hilbert space spanned by the vectors $| \pm n\rangle=\prod_{n} \hat{p}_{ \pm}^{n}|0\rangle$. Employing this basis the correlators of the bulk field $\hat{O}$ of mass $m$ and dimension $\Delta=(d+1) / 2 \pm l m$ can be evaluated 
by the saddle point method, thereby resulting in the semiclassical expression for the (real-time) two-point function

$$
\begin{aligned}
& G_{O O}(x, p, t) \sim \\
& \exp \left(-\Delta \int\left[g_{p p} \mathrm{~d} p^{2}-g_{t t} \mathrm{~d} t^{2}+g_{x x} \mathrm{~d} x^{2}\right]^{1 / 2}\right),
\end{aligned}
$$

where the line integral is taken over the $3 d$ geodesic connecting the end points.

Placing the end points of this correlation function on the boundary yields the single-particle boundary propagator. Fourier transforming this expression in the spacetime domain one then obtains

$$
\begin{aligned}
& G_{O O}(\omega, k) \sim \\
& \exp \left(-\int \mathrm{d} p\left[q_{p p}\left(\frac{\Delta^{2}}{l^{2}}-\frac{\omega^{2}}{g_{t t}}+\frac{k^{2}}{g_{x x}}\right)\right]^{1 / 2}\right) .
\end{aligned}
$$

For instance, the BTZ bulk metric (40) yields the following propagator of massless $3 d$ bulk fermions with the dimension $\Delta_{\Psi}=1$ and spin $1 / 2$ [84, 171-182,

$$
G_{\Psi \Psi}^{ \pm}(\omega, k)=\left(\frac{\omega \pm k}{\omega \mp k}\right)^{1 / 2},
$$

which gives rise to the power-law spacetime behaviour of the boundary propagator $G_{\Psi \psi}^{ \pm}(t, x) \sim$ $1 /\left|x_{ \pm}\right|^{2}$. These results should, of course, be distinguished from the standard LL propagator $G_{\Psi \Psi}^{ \pm}(\omega, k)=1 /(\omega \mp k)$ of free chiral fermions with the dimension $\Delta_{\psi}=1 / 2$.

In contrast, using the metric build out of the solitonic solution (29) changes the spacetime decay from algebraic to exponential, $G_{\Psi \Psi}^{ \pm}(t, x) \sim$ $\exp \left(-\Delta\left|x_{ \pm}\right| / l\right)$. In turn, the two-particle (energy) excitations representing gapless boundary gravitons remain propagating, thus featuring the ordinary ballistic pole

$$
G_{\epsilon \epsilon}(\omega, k)=\int \mathrm{d} t \mathrm{~d} x \mathrm{e}^{\mathrm{i}(k x-\omega t)}\left[G_{\psi \psi}^{ \pm}(x, t)\right]^{2} \sim \frac{k^{2}}{k^{2}-\omega^{2}} .
$$

Alternatively, this energy correlation function can be obtained from the correlator $\langle w(x, p, t) \times$ $\left.w\left(x^{\prime}, p^{\prime}, t^{\prime}\right)\right\rangle$ computed as the path integral over the Wigner function.

The ballistic behaviour (48) should be contrasted against the diffusive one observed in, e.g. a chain of coupled SYK models, $G_{\epsilon \epsilon}(\omega, k) \sim k^{2} /\left(k^{2}+\mathrm{i} \omega\right)$, which would be indicative of a (maximally) chaotic state [132-170].

Further possible generalizations of the collective field hydrodynamics include incorporation of the momentum Berry curvature in Eq. (6), exploration of the effects of viscosity, generic dispersion with $z \neq 1,2$, etc. It would also be interesting to investigate a development of turbulence which has long been known to harbour some important connections to quantum gravity.

\section{Discussion}

In this note, the Wigner function representation of generic quantum systems was discussed from the standpoint of pinpointing the possible origin of the hypothetical generalized holographic correspondence. To that end, using the Kirillov-Kostant procedure of phase space quantization via the coherent state path integral and collective field hydrodynamics may seem rather promising.

Specifically, in line with the holographic lore, path integral quantization on a co-adjoint orbit of the $W_{\infty}$ group of the volume-preserving diffeomorphisms of the phase space exposes an intrinsic relationship between the $2 d+1$-dimensional 'bulk' description and the $d+1$-dimensional 'boundary' hydrodynamics. The quantum bulk dynamics is described by the action composed of the $W_{\infty}$ generators while the corresponding boundary variables are given by the moments (17) of the Wigner function. Systematically implementing this program can then be thought of as 'deriving' the sought-out holographic duality.

Importantly, such a generic form of correspondence neither requires a reference to some underlying 'string' theory, nor does it impose any particular symmetry conditions on the boundary system in question, while the putative bulk description does not necessarily have a gravity-like appearance.

Nonetheless, in the case of $d=1$ the corresponding $3 d$ bulk behaviour can indeed be cast in terms of the (doubled) Chern-Simons theory or, equivalently, non-dynamical Einstein gravity with a negative cosmological constant. Furthermore, if the boundary Hamiltonian belongs to the integrable (e.g. KdV) family, the corresponding set of the bulk metrics may include the familiar 
BTZ, as would be generally anticipated in line with the AdS/CFT paradigm [1-7].

Furthermore, in $d=1$ the phase space description can be viewed as a formally exact (non-linear) bosonization of the boundary system. Many of such systems appear to be integrable (hence, nonergodic) and possess an infinite number of locally conserved currents given by the various moments of the Wigner function which obey the equations of zero-entropy generalized quantum hydrodynamics of the GGE type.

In higher dimensions the moments of Wigner function also serve as coefficients in the series expansions of the would-be local bulk metrics over the powers of the momentum p. Although with the increasing spatial dimension the hydrodynamic description becomes less accurate, it remains capable of capturing the salient features of the quantum phase space dynamics governed by the conservation laws.

To summarize, the use of the phase space approach brings out the intrinsic correspondence between formally exact $2 d+1$-dimensional and less accurate ('coarse-grained') $d+1$-dimensional hydrodynamic descriptions of a given quantum system. In this general setting, neither the latter needs to be a conformal field theory, nor does the former have to necessarily have the appearance of classical gravity.

In those $d=0$ and $d=1$ cases, where the bulk indeed appears to be amenable to a gravitational description, the gravity theory has no dynamics of its own and is fully determined by the boundary degrees of freedom. Accordingly, the viable bulk metrics can be mapped onto the solutions of the boundary hydrodynamics.

In that regard, the holographic custom of picking out a particular metric and claiming some sort of the Einstein-Maxwell-dilaton theory to be the proper bulk dual of a certain strongly correlated system does not appear to be backed by the above conclusions. Nevertheless, in some cases, including $d=0$ and $d=1$, certain phenomenological predictions may indeed turn out to be right - albeit, quite possibly, for the wrong reason.

However, if all the essentials of practical holography were to amount to little else but hydrodynamics - as the above discussion may seem to suggest - then the whole issue of its justification and validity would be largely moot and void.

\section{References}

[1] S.A. Hartnoll, Lectures on holographic methods for condensed matter physics, Class. Quant. Grav. 26, 224002 (2009).

[2] C.P. Herzog, Lectures on holographic superfluidity and superconductivity, J. Phys. A 42, 343001 (2009).

[3] J. McGreevy, Holographic duality with a view toward many-body physics, Adv. High Energy Phys. 2010, 723105 (2010).

[4] S. Sachdev, What can gauge-gravity duality teach us about condensed matter physics? Annu. Rev. Cond. Matt. Phys. 3, 9-33 (2012).

[5] J. Zaanen. Y. Liu, Y.-W. Sun, and K. Schalm, Holographic Duality in Condensed Matter Physics (Cambridge University Press, 2015).

[6] M. Ammon and J. Erdmenger, Gauge/Gravity Duality (Cambridge University Press, 2015).

[7] S.A. Hartnoll, A. Lucas, and S. Sachdev, Holographic Quantum Matter (MIT Press, 2018).

[8] https://arXiv.org/search/?query=holographic+co ndmat

[9] https://projects.ift.uam-csic.es/holotube/videogallery/

[10]A. Dhar, G. Mandal, and S.R. Wadia, Stringy quantum effects in two-dimensional black hole, Mod. Phys. Lett. A 7, 3703-3715 (1992), arXiv:hep-th/9210120

[11]A. Dhar, G. Mandal, and S.R. Wadia, Classical Fermi fluid and geometric action for $c=1$, Int. J. Mod. Phys. A 8(2), 325-350 (1993), arXiv:hepth/9204028

[12]A. Dhar, G. Mandal, and S.R. Wadia, Nonrelativistic fermions, coadjoint orbits of $W_{\infty}$ and string field theory at $c=1$, Mod. Phys. Lett. A 7, 3129-3146 (1992), arXiv:hep-th/9207011

[13]A. Dhar, G. Mandal, and S.R. Wadia, $W_{\infty}$ coherent states and path-integral derivation of bosonization of non-relativistic fermions in one dimension, Mod. Phys. Lett. A 8, 3557-3568 (1993), arXiv:hepth/9309028

[14]A. Dhar, G. Mandal, and S.R. Wadia, A timedependent classical solution of $c=1$ string field theory and nonperturbative effects, Int. J. Mod. Phys. A 8, 3811-3828 (1993), arXiv:hep-th/ 9212027 
[15]S.R. Das, A. Dhar, G. Mandal, and S.R. Wadia, Bosonization of nonrelativistic fermions and Winfinity algebra, Mod. Phys. Lett. A 7, 71-84 (1992), arXiv:hep-th/9111021

[16]S.R. Das, A. Dhar, G. Mandal, and S.R. Wadia, $W$-infinity Ward identities and correlation functions in the $C=1$ matrix model, Mod. Phys. Lett. A 7, 937-954 (1992); Erratum-ibid. A7, 2245 (1992), arXiv:hep-th/9112052

[17]S.R. Das, D branes in $2 \mathrm{~d}$ string theory and classical limits, arXiv:hep-th/0401067

[18]S.R. Das, Non-trivial 2d space-times from matrices, arXiv:hep-th/0503002

[19]S. Iso, D. Karabali, and B. Sakita, One-dimensional fermions as two-dimensional droplets via Chern-Simons theory, Nucl. Phys. B 388, 700714 (1992), arXiv:hep-th/9202012

[20]S.R. Das and L.H. Santos, Open string descriptions of spacelike singularities in two dimensional string theory, Phys. Rev. D 75, 126001 (2007), arXiv:hepth/0702145

[21]N. Banerjee and S. Dutta, Holographic hydrodynamics: models and methods, arXiv: 1112.5345

[22]S.R. Das and S.D. Mathur, Folds, bosonization and non-triviality of the classical limit of $2 \mathrm{D}$ string theory, Phys. Lett. B 365, 79-86 (1996), arXiv:hepth/9507141

[23]A. Jevicki, in: String Theory, Gauge Theory and Quantum Gravity '93, Proceedings of the Trieste Spring School and Workshop, ICTP, Trieste, Italy, 19-29 April 1993 (World Scientific, 2014) pp. 96140, arXiv:hepth/9309115

[24]A. Luther, Tomonaga fermions and the Dirac equation in three dimensions, Phys. Rev. B 19, 320 (1979).

[25]F.D.M. Haldane, in: Proceedings of the International School of Physics 'Enrico Fermi', Course CXXI 'Perspectives in Many-Particle Physics', eds. R.A. Broglia and J.R. Schrieffer (North-Holland, Amsterdam 1994) pp.5-29, arXiv:cond-mat/ 0505529

[26]D.V. Khveshchenko and P.C.E. Stamp, Lowenergy properties of two-dimensional fermions with long-range current-current interactions, Phys. Rev. Lett. 71, 2118 (1993).
[27]D.V. Khveshchenko and P.C.E. Stamp, Eikonal approximation in the theory of two-dimensional fermions with long-range current-current interactions, Phys. Rev. B 49(8), 5227 (1994).

[28]A.C. Neto and E. Fradkin, Bosonization of the low energy excitations of Fermi liquids, Phys. Rev. Lett 72, 1393 (1994).

[29]A.C. Neto and E. Fradkin, Bosonization of Fermi liquids, Phys. Rev. B 49, 10877 (1994).

[30]A. Hougton and J.B. Marston, Bosonization and fermion liquids in dimensions greater than one, Phys. Rev. B 48, 7790 (1993).

[31]A. Hougton, H.J. Kwon, and J.B. Marston, Gauge interactions and bosonized fermion liquids, Phys. Rev. Lett. 73, 284 (1994).

[32]A. Hougton, H.J. Kwon, and J.B. Marston, Stability and single-particle properties of bosonized Fermi liquids, Phys. Rev. B 50, 1351 (1994).

[33]A. Hougton, H.J. Kwon, and J.B. Marston, Multidimensional bosonization, Adv. Phys. 49, 141-228 (2000).

[34]D.V. Khveshchenko, R. Hlubina, and T.M. Rice, Non-Fermi-liquid behaviour in two dimensions due to long-ranged current-current interactions, Phys. Rev. B 48, 10766 (1993).

[35]L.B. Ioffe, D. Lidsky, and B.L. Altshuler, Effective lowering of dimensionality in the strongly correlated two dimensional electron gas, Phys. Rev. Lett. 73, 472 (1994).

[36]C. Castellani, C. Di Castro, and W. Metzner, Dimensional crossover from Fermi to Luttinger liquid, Phys. Rev. Lett. 72, 316 (1994).

[37]J. Polchinski, Low-energy dynamics of the spinon-gauge system, Nucl. Phys. B 422, 617 (1994).

[38]D.V. Khveshchenko, Bosonization of currentcurrent interactions, Phys. Rev. B 49, 16893 (1994).

[39]D.V. Khveshchenko, Towards exact bosonization of the Calogero-Sutherland model, Int. J. Modern Phys. B 9(13-14), 1639-1646 (1995), arXiv:condmat/9404094

[40]D.V. Khveshchenko, Geometrical approach to bosonization of $D>1$ dimensional (non)-Fermi liquids, Phys. Rev. B 52, 4833 (1995). 
[41]A.M.J. Schakel, Effective field theory of idealfluid hydrodynamics, Mod. Phys. Lett. B 10, 999 (1996), arXiv:cond-mat/9607164

[42]A.G. Abanov and P.B. Wiegmann, Quantum hydrodynamics, the quantum Benjamin-Ono equation, and the Calogero model, Phys. Rev. Lett. 95, 076402 (2005), arXiv:cond-mat/0504041

[43]E. Bettelheim, A.G. Abanov, and P. Wiegmann, Nonlinear dynamics of quantum systems and soliton theory, J. Phys. A 40, F193-F208 (2007), arXiv:nlin/0605006

[44]A.G. Abanov, E. Bettelheim, and P. Wiegmann, Integrable hydrodynamics of Calogero-Sutherland model: bidirectional Benjamin-Ono equation, J. Phys. A 42, 135201 (2009), arXiv: 0810.5327

[45]E. Bettelheim, A.G. Abanov, and P. Wiegmann, Quantum hydrodynamics and nonlinear differential equations for degenerate Fermi gas, J. Phys. A 41, 392003 (2008), arXiv:0804.2272

[46]E. Bettelheim, A.G. Abanov, and P. Wiegmann, Nonlinear quantum shock waves in fractional quantum Hall edge states, Phys. Rev. Lett. 97, 246402 (2006), arXiv:cond-mat/0607453

[47]P.B. Wiegmann, Nonlinear hydrodynamics and fractionally quantized solitons at the fractional quantum Hall edge, Phys. Rev. Lett. 108, 206810 (2012), arXiv:1112.0810

[48]P.B. Wiegmann, Quantum hydrodynamics of fractional Hall effect: Quantum Kirchhoff equations, arXiv:1211.5132

[49]E. Bettelheim and P. Wiegmann, Universal Fermi distribution of semiclassical nonequilibrium Fermi states, Phys. Rev. B 84, 085102 (2011), arXiv: 1104.1854

[50]E. Bettelheim, Y. Kaplan, and P. Wiegmann, Fermi edge resonances in non-equilibrium states of Fermi gases, J. Phys. A 44, 282001 (2011), arXiv: 1103.4236

[51]E. Bettelheim, Y. Kaplan, and P. Wiegmann, Gradient catastrophe and Fermi-edge resonances in Fermi gas, Phys. Rev. Lett. 106, 166804 (2011), arXiv:1011.1993

[52]M. Laskin, T. Can, and P. Wiegmann, Collective field theory for quantum Hall states, Phys. Rev. B 92, 235141 (2015), arXiv:1412.8716
[53] M. Laskin, Y.H. Chiu, T. Can, and P. Wiegmann, Emergent conformal symmetry of quantum Hall states on singular surfaces, Phys. Rev. Lett. 117, 266803 (2016), arXiv:1602.04802

[54]S. Klevtsov, X. Ma, G. Marinescu, and P. Wiegmann, Quantum Hall effect and Quillen metric, Commun. Math. Phys. 349, 819-855 (2017), arXiv:1510.06720

[55]M. Pustilnik and K.A. Matveev, Fate of classical solitons in one-dimensional quantum systems, Phys. Rev. B 92, 195146 (2015), arXiv:1507.05639

[56]M. Pustilnik and K.A. Matveev, Viscous dissipation in one-dimensional quantum liquids, Phys. Rev. Lett. 119, 036801 (2017), arXiv:1706.07004

[57]M. Pustilnik and K.A. Matveev, Effective mass of elementary excitations in Galilean-invariant integrable models, Phys. Rev. B 94, 115436 (2016), arXiv:1606.05553

[58]B. Doyon, H. Spohn, and T. Yoshimura, A geometric viewpoint on generalized hydrodynamics, Nucl. Phys. B 926, 570-583 (2017), arXiv: 1704.04409

[59]B. Doyon and T. Yoshimura, A note on generalized hydrodynamics: inhomogeneous fields and other concepts, SciPost Phys. 2, 014 (2017), arXiv: 1611.08225

[60]M. Fagotti, Higher-order generalized hydrodynamics in one dimension: The noninteracting test, Phys. Rev. B 96, 220302 (2017), arXiv:1708.05383

[61]A. Bastianello, B. Doyon, G. Watts, and T. Yoshimura, Generalized hydrodynamics of classical integrable field theory: the sinh-Gordon model, SciPost Phys. 4, 045 (2018), arXiv: 1712.05687

[62]B. Doyon, Lecture notes on generalised hydrodynamics, SciPost Phys. Lect. Notes 18 (2020), arXiv:1912.08496

[63]P. Ruggiero, P. Calabrese, B. Doyon, and J. Dubail, Quantum generalized hydrodynamics, Phys. Rev. Lett. 124, 140603 (2020), arXiv.org: 1910.00570

[64]Z.-Y. Shi, C. Gao, and H. Zhai, Idealized hydrodynamics, arXiv:2011.01415

[65]D.S. Dean, P. Le Doussal, S.N. Majumdar, and G. Schehr, Nonequilibrium dynamics of noninteracting fermions in a trap, EPL 126, 20006 (2019), arXiv:1902.02594 
[66]D.S. Dean, P. Le Doussal, S.N. Majumdar, and G. Schehr, Impurities in systems of noninteracting trapped fermions, SciPost Phys. 10, 082 (2021), arXiv:2012.13958

[67]H. Spohn, Hydrodynamic equations for the Toda lattice, arXiv:2101.06528

[68]J.R. Klauder, in: Contemporary Problems in Mathematical Physics, Proceedings of the Second International Workshop, Cotonou, Republic of Benin, 28 October - 2 November 2001, eds. J. Govaerts and A.Z. Msezane (World Scientific, 2002) pp. 395-408, arXiv:quant-ph/0112010

[69]C. Anastopoulos, Generalized coherent states for spinning relativistic particles, J. Phys. A 37, 8619 (2004), arXiv:quant-ph/0312025

[70]T. Curtright and D. Fairlie, Extra dimensions and nonlinear equations, J. Math. Phys. 44, 26922703 (2003), arXiv:math-ph/0207008

[71]T. Curtright and D. Fairlie, Morphing quantum mechanics and fluid dynamics, J. Phys. A 36, 8885-8902 (2003), arXiv:math-ph/0303003

[72]R. Carroll, Remarks on the Schrodinger equation, arXiv:quant-ph/0401082

[73]D.H. Delphenich, The geometric origin of the Madelung potential, arXiv:gr-qc/0211065

[74]T. Curtright, d-branes in the stream, arXiv:hepth/0307121

[75] C. Anastopoulos and N. Savvidou, The role of phase space geometry in Heisenberg's uncertainty relation, Annals Phys. 308, 329-353 (2003), arXiv:quantph/0304049

[76]T.L. Curtright and C.K. Zachos, Quantum mechanics in phase space, APPN 1(1), 37-46 (2012), arXiv:1104.5269

[77]V.V. Dodonov and A.V. Dodonov, Energy-time and frequency-time uncertainty relations: exact inequalities, arXiv:1504.00862

[78]M. Geiller, C. Goeller, and N. Merino, Most general theory of $3 \mathrm{~d}$ gravity: covariant phase space, dual diffeomorphisms, and more, JHEP 2, 120 (2021), arXiv:2011.09873

[79]A. Achúcarro and P.K. Townsend, A ChernSimons action for three-dimensional anti-de Sitter supergravity theories, Phys. Lett. B 180, 89 (1986).
[80]E. Witten, Quantum field theory and the Jones polynomial, Commun. Math. Phys. 121, 351 (1989).

[81]J.D. Brown and M. Henneaux, Central charges in the canonical realization of asymptotic symmetries: An example from three dimensional gravity, Commun. Math. Phys. 104, 207 (1986).

[82]O. Coussaert, M. Henneaux, and P. van Driel, The asymptotic dynamics of three-dimensional Einstein gravity with a negative cosmological constant, Class. Quant. Grav. 12, 2961-2966 (1995), arXiv:grqc/9506019.

[83]R.E. Goldstein and D.M. Petrich, The Kortewegde Vries hierarchy as dynamics of closed curves in the plane, Phys. Rev. Lett. 67, 1991 (3203).

[84]H. Afshar, S. Detournay, D. Grumiller, and B. Oblak, Near-horizon geometry and warped conformal symmetry, JHEP 2016, 187 (2016), arXiv: 1512.08233

[85]H. Afshar, D. Grumiller, W. Merbis, A. Perez, D. Tempo, and R. Troncoso, Soft hairy horizons in three spacetime dimensions, Phys. Rev. D 95, 106005 (2017), arXiv:1611.09783

[86]H. Afshar, S. Detournay, D. Grumiller, W. Merbis, A. Perez, D. Tempo, and R. Troncoso, Soft Heisenberg hair on black holes in three dimensions, Phys. Rev. D 93, 101503 (2016), arXiv: 1603.04824

[87]M. Henneaux, W. Merbis, and A. Ranjbar, Asymptotic dynamics of $\mathrm{AdS}_{3}$ gravity with two asymptotic regions, JHEP 2020, 64 (2020), arXiv: 1912.09465

[88]S. Li, N. Toumbas, and J. Troost, Liouville quantum gravity, Nucl. Phys. B 952, 114913 (2020), arXiv:1903.06501

[89]A. Pérez, D. Tempo, and R. Troncoso, Boundary conditions for General Relativity on AdS3 and the KdV hierarchy, JHEP 2016, 103 (2016), arXiv: 1605.04490

[90]O. Fuentealba, J. Matulich, A. Pérez, M. Pino, P. Rodríguez, D. Tempo, and R. Troncoso, Integrable systems with BMS3 Poisson structure and the dynamics of locally flat spacetimes, JHEP 2018, 148 (2018), arXiv:1711.02646 
[91]D. Grumiller and W. Merbis, Near horizon dynamics of three dimensional black holes, SciPost Phys. 8, 010 (2020), arXiv:1906.10694

[92]E. Ojeda and A. Pérez, Boundary conditions for General Relativity in three-dimensional spacetimes, integrable systems and the $\mathrm{KdV} /$ mKdV hierarchies, JHEP 2019, 79 (2019), arXiv:1906.11226

[93]A. Dymarsky and S. Sugishita, KdV-charged black holes, JHEP 2020, 41 (2020), arXiv:2002.08368

[94]J. Matulich, S. Prohazka, and J. Salzer, Limits of three-dimensional gravity and metric kinematical Lie algebras in any dimension, JHEP 2019, 118 (2019), arXiv:1903.09165

[95]J. Cotler and K. Jensen, AdS 3 gravity and random CFT, JHEP 2021, 33 (2021), arXiv:2006.08648

[96]M. Henneaux and S.-J. Rey, Nonlinear W $\infty$ as asymptotic symmetry of three-dimensional higher spin AdS gravity, JHEP 2010, 7 (2010), arXiv:1008.4579

[97]G. Compère and W. Song, W symmetry and integrability of higher spin black holes, JHEP 2013, 144 (2013), arXiv:1306.0014

[98]M. Gutperle and Y. Li, Higher spin Lifshitz theory and integrable systems, Phys. Rev. D 91, 046012 (2015), arXiv:1412.7085

[99] M. Beccaria, M. Gutperle, Y. Li, and G. Macorini, Higher spin Lifshitz theories and the Korteweg-de Vries hierarchy, Phys. Rev. D 92, 085005 (2015), arXiv:1504.06555

[100]D. Grumiller, A. Pérez, S. Prohazka, D. Tempo, and R. Troncoso, Higher spin black holes with soft hair, JHEP 10, 119 (2016), arXiv:1607.05360

[101]E. Ojeda and A. Pérez, Integrable systems and the boundary dynamics of higher spin gravity on $\mathrm{AdS}_{3}$, JHEP 2020, 89 (2020), arXiv:2009.07829

[102]P.M. Petropoulos, Gravitational duality, topologically massive gravity and holographic fluids, arXiv:1406.2328

[103]P.M. Petropoulos and K. Siampos, Integrability, Einstein spaces and holographic fluids, arXiv: 1510.06456

[104]J. Gath, A. Mukhopadhyay, A.C. Petkou, P.M. Petropoulos, and K. Siampos, Petrov classification and holographic reconstruction of spacetime, JHEP 2015, 5 (2015), arXiv:1506.04813
[105]L. Ciambelli, C. Marteau, A.C. Petkou, P.M. Petropoulos, K. Siampos, Covariant Galilean versus Carrollian hydrodynamics from relativistic fluids, Class. Quantum Grav. 35, 165001 (2018), arXiv:1802.05286

[106]L. Ciambelli, C. Marteau, A.C. Petkou, P.M. Petropoulos, and K. Siampos, Flat holography and Carrollian fluids, JHEP 1807, 165 (2018), arXiv:1802.06809

[107]D. Hansen, J. Hartong, and N.A. Obers, Nonrelativistic gravity and its coupling to matter, JHEP 2020, 145 (2020), arXiv:2001.10277

[108]J. de Boer and D. Engelhardt, Remarks on thermalization in 2D CFT, Phys. Rev. D 94, 126019 (2016), arXiv:1604.05327

[109]M. Rangamani, S.F. Ross, D.T. Son, and E.G. Thompson, Conformal non-relativistic hydrodynamics from gravity, JHEP 2009, 075 (2009), arXiv:0811.2049

[110]A. Campoleoni, L. Ciambelli, C. Marteau, P.M. Petropoulos, and K. Siampos, Twodimensional fluids and their holographic duals, Nucl. Phys. B 946, 114692 (2019), arXiv: 1812.04019

[111]M. Kulkarni, G. Manda, and T. Morita, Quantum quench and thermalization of onedimensional Fermi gas via phase-space hydrodynamics, Phys. Rev. A 98, 043610 (2018), arXiv:1806.09343

[112]S.R. Das, S. Hampton, and S. Liu, Quantum quench in $c=1$ matrix model and emergent spacetimes, JHEP 2020, 107 (2020), arXiv: 1910.00123

[113]S.R. Das, S. Hampton, and S. Liu, Quantum quench in non-relativistic fermionic field theory: Harmonic traps and 2d string theory, JHEP 2019, 176 (2019), arXiv:1903.07682

[114]S. Bhattacharyya, V.E. Hubeny, S. Minwalla, and M. Rangamani, Nonlinear fluid dynamics from gravity, JHEP 2008, 045 (2008), arXiv:0712.2456

[115]S. Bhattacharyya, V.E. Hubeny, R. Loganayagam, G. Mandal1, S. Minwalla, T. Morita, M. Rangamani, and HS. Reall, Local fluid dynamical entropy from gravity, JHEP 2008(6), 055 (2008), arXiv:0803.2526 
[116]N. Ambrosetti, J. Charbonneau, and S. Weinfurtner, The fluid/gravity correspondence: Lectures notes from the 2008 Summer School on Particles, Fields, and Strings, arXiv:0810.2631

[117]N. Banerjee, J. Bhattacharya, S. Bhattacharyya, S. Dutta, R. Loganayagam, and P. Surówka, Hydrodynamics from charged black branes, JHEP 2011, 094 (2011), arXiv:0809.2596

[118]S. Bhattacharyya, S. Minwalla, and S.R. Wadia, The incompressible non-relativistic NavierStokes equation from gravity, JHEP 2009, 59 (2009), arXiv:0810.1545

[119] M. Haack and A. Yarom, Nonlinear viscous hydrodynamics in various dimensions using AdS/ CFT, JHEP 2008, 63 (2008), arXiv:0806.4602

[120]S. Bhattacharyya, R. Loganayagam, I. Mandal, S. Minwalla, and A. Sharma, Conformal nonlinear fluid dynamics from gravity in arbitrary dimensions, JHEP 2008(12), 116 (2008), arXiv: 0809.4272

[121]M. Rangamani, Gravity and hydrodynamics: lectures on the fluid-gravity correspondence, Class. Quant. Grav. 26, 224003 (2009), arXiv: 0905.4352

[122]V.E. Hubeny, S. Minwalla, and M. Rangamani, in: Black Holes in Higher Dimensions (Cambridge University Press, 2012) pp. 348-386, arXiv: 1107.5780

[123]N. Banerjee and S. Dutta, Holographic hydrodynamics: models and methods, arXiv:1112.5345

[124]Y. Bu and M. Lublinsky, Linearized fluid/gravity correspondence: from shear viscosity to all order hydrodynamics, JHEP 2014, 64 (2014), arXiv:1409.3095

[125]L. Ciambelli, C. Marteau, P.M. Petropoulos, and R. Ruzziconi, Fefferman-Graham and Bondi gauges in the fluid/gravity correspondence, arXiv:2006.10083

[126]G. Barnich, H.A. González, and P. SalgadoRebolledo, Geometric actions for three-dimensional gravity, Class. Quantum Grav. 35, 014003 (2018), arXiv:1707.08887

[127]P. Caputa and J.M. Magan, Quantum computation as gravity, Phys. Rev. Lett. 122, 231302 (2019), arXiv:1807.04422
[128]J.M. Magan, Black holes, complexity and quantum chaos, JHEP 2018, 43 (2018), arXiv: 1805.05839

[129]J. Ergmenter, M. Gerbershagen, and A.-L. Weigel, Complexity measures from geometric actions on Virasoro and Kac-Moody orbits, JHEP 2020(11), 003 (2020), arXiv:2004.03619

[130]A. Jain, Effective field theory for non-relativistic hydrodynamics, JHEP 2020, 208 (2020), arXiv: 2008.03994

[131]W. Merbis and M. Riegler, Geometric actions and flat space holography, JHEP 2020, 125 (2020), arXiv:1912.08207

[132]S. Sachdev and J. Ye, Gapless spin-fluid ground state in a random quantum Heisenberg magnet, Phys. Rev. Lett. 70, 3339 (1993).

[133]S. Sachdev, Holographic metals and the fractionalized Fermi liquid, Phys. Rev. Lett. 105, 151602 (2010).

[134]S. Sachdev, Bekenstein-Hawking entropy and strange metals, Phys. Rev. X 5, 041025 (2015).

[135]A. Kitaev, KITP Seminars, 2015, http://online. kitp.ucsb.edu,

[136] A. Kitaev, Notes on $\widetilde{S} L(2$,$) representations, arXiv:$ 1711.08169

[137]A. Kitaev and S.J. Suh, The soft mode in the SachdevYe-Kitaev model and its gravity dual, JHEP 2018, 183 (2018), arXiv:1711.08467

[138]A. Kitaev and S.J. Suh, Statistical mechanics of a two-dimensional black hole, JHEP 2019, 198 (2019), arXiv:1808.07032

[139]E. Witten, An SYK-Like model without disorder, arXiv:1610.09758

[140]R. Gurau, The complete $1 / N$ expansion of a SYKlike tensor model, Nucl. Phys. B 916, 386-401 (2017), arXiv:1611.04032

[141]R. Gurau, The prescription in the SYK model, arXiv:1705.08581

[142]I.R. Klebanov and G. Tarnopolsky, Uncolored random tensors, melon diagrams, and the Sachdev-Ye-Kitaev models, Phys. Rev. D 95, 046004 (2017).

[143]S. Giombi, I.R. Klebanov, and G. Tarnopolsky, Bosonic tensor models at large $N$ and small $\varepsilon$, Phys. Rev. D 96, 106014 (2017). 
[144]J. Maldacena, S.H. Shenker, and D. Stanford, A bound on chaos, JHEP 2016(8), 106 (2016).

[145]J. Maldacena and D. Stanford, Remarks on the Sachdev-Ye-Kitaev model, Phys. Rev. D 94, 106002 (2016).

[146]J. Maldacena, D. Stanford, and Z. Yang, Conformal symmetry and its breaking in two dimensional nearly Anti-de-Sitter space, arXiv: 1606.01857

[147]D. Stanford and E. Witten, Fermionic localization of the schwarzian theory, JHEP 2017(10), 8 (2017).

[148]J. Polchinski and V. Rosenhaus, The spectrum in the Sachdev-Ye-Kitaev model, JHEP 2016(4), 1 (2016).

[149]D.J. Gross and V. Rosenhaus, The bulk dual of SYK: cubic couplings, JHEP 2017(05), 92 (2017).

[150]D.J. Gross and V. Rosenhaus, All point correlation functions in SYK, JHEP 2017(12), 148 (2017).

[151]G. Sárosi, in: XIII Modave Summer School in Mathematical Physics (Modave2017), Proc. Sci. Vol. 323, arXiv:1711.08482

[152]H.W. Lin, J. Maldacena, and Y. Zhao, Symmetries near the horizon, JHEP 2019, 49 (2019), arXiv: 1904.12820

[153]Y. Gu, X.-L. Qi, and D. Stanford, Local criticality, diffusion and chaos in generalized SachdevYe-Kitaev models, JHEP 2017, 125 (2017).

[154]Y. Gu, A. Lucas, and X.-L. Qi, Energy diffusion and the butterfly effect in inhomogeneous Sachdev-Ye-Kitaev chains, SciPost Phys. 2, 018 (2017).

[155]Y. Gu, A. Lucas, and X.-L. Qi, Spread of entanglement in a Sachdev-Ye-Kitaev chain, JHEP 2017, 120 (2017).

[156]Y. Gu and A. Kitaev, On the relation between the magnitude and exponent of OTOCs, JHEP 2019(2), 75 (2019).

[157]D. Bagrets, A. Altland and A. Kamenev, Sachdev-Ye-Kitaev model as Liouville quantum mechanics, Nucl. Phys. B 911, 191-205 (2016).

[158]D. Bagrets, A. Altland and A. Kamenev, Powerlaw out of time order correlation functions in the SYK model, Nucl. Phys. B 921, 727 (2017).
[159]T.G. Mertens, G.J. Turiaci, and H.L. Verlinde, Solving the Schwarzian via the conformal bootstrap, JHEP 8, 136 (2017).

[160]T.G. Mertens, The Schwarzian theory - origins, JHEP 2018(5), 36 (2018).

[161]Z. Yang, The quantum gravity dynamics of near extremal black holes, JHEP 2019(5), 205 (2019), arXiv: 1809.08647

[162]A. Jevicki, K. Suzuki, and J. Yoon, Bi-local holography in the SYK model, JHEP 2016(7), 7 (2016).

[163]A. Jevicki and K. Suzuki, Bi-local holography in the SYK model: Perturbations, arXiv: 1608.07567

[164]S.R. Das, A. Jevicki, and K. Suzuki, Three dimensional view of the SYK/AdS duality, JHEP 2017(9), 17 (2017).

[165]S.R. Das, A. Ghosh, A. Jevicki, and K. Suzuki, Spacetime in the SYK model, JHEP 2018(7), 184 (2018).

[166]S.R. Das, A. Ghosh, A. Jevicki, and K. Suzuki, Three dimensional view of arbitrary q SYK models, JHEP 2018(2), 162 (2018).

[167]G. Mandal, P. Nayak, and S.R. Wadia, Coadjoint orbit action of Virasoro group and two-dimensional quantum gravity dual to SYK/tensor models, JHEP 2017(11), 46 (2017).

[168]A. Gaikwad, L. Kh Joshi, G. Mandal, and S.R. Wadia, Holographic dual to charged SYK from 3D Gravity and Chern-Simons, JHEP 2020(2), 33 (2020), arXiv:1802.07746

[169]T.G. Mertens and G.J. Turiaci, Defects in JackiwTeitelboim quantum gravity, JHEP 2019(8), 127 (2019), arXiv:1904.05228

[170]D.V. Khveshchenko, On a (pseudo)holographic nature of the SYK-like models, Lith. J. Phys. 59, 104 (2019), arXiv:1905.04381

[171]V. Balasubramanian, I. García-Etxebarria, F. Larsen, and J. Simón, Helical Luttinger liquids and three dimensional black holes, Phys. Rev. D 84, 126012 (2011), arXiv:1012.4363

[172]D. Maity, S. Sarkar, B. Sathiapalan, R. Shankar, and N. Sircar, Properties of CFTs dual to charged BTZ black-hole, Nucl. Phys. B 839, 526-551 (2010), arXiv:0909.4051 
[173]B. Lian, S.L. Sondhi, and Z. Yang, The chiral SYK model, JHEP 2019(9), 067 (2019), arXiv: 1906.03308

[174]M. Banados, Three-dimensional quantum geometry and black holes, AIP Conf. Proc. 484, 147 (1999), arXiv:hep-th/9901148

[175]R. Li and J.-R. Ren, Dirac particles tunneling from BTZ black hole, Phys. Lett. B 661, 370372 (2008), arXiv:0802.3954

[176]N. Iqbal and H. Liu, Real-time response in AdS/ CFT with application to spinors, Fortsch. Phys. 57, 367-384 (2009), arXiv:0903.2596

[177]A. Dasgupta, Emission of fermions from BTZ black holes, Phys. Lett. B 445, 279-286 (1999), arXiv:hepth/9808086

[178]D. Maity, S. Sarkar, B. Sathiapalan, R. Shankar, and N. Sircar, Properties of CFTs dual to charged BTZ black hole, Nucl. Phys. B 839, 526-551 (2010), arXiv:0909.4051

[179]S.-W. Zhou, X.-X. Zeng, and W.-B. Liu, Hawking radiation from a BTZ black hole viewed as Landauer transport, arXiv:1106.0559

[180]L.G.C. Gentile, P.A. Grassi, and A. Mezzalira, Fermionic corrections to fluid dynamics from BTZ black hole, JHEP 2015, 153 (2015), arXiv: 1302.5060

[181]T. Faulkner and N. Iqbal, Friedel oscillations and horizon charge in 1D holographic liquids, arXiv:1207.4208

[182]L.-Y. Hung and A. Sinha, Holographic quantum liquids in 1+1 dimensions, JHEP 2010(1), 114 (2010), arXiv:0909.3526

\section{PAPRASČIAUSIA FAZINĖS ERDVĖS HOLOGRAFIJA}

D.V. Khveshchenko

Šiaurès Karolinos universitetas, Čepel Hilas, JAV 